\title{
Données complémentaires sur la systématique et l'écologie de Siettitia avenionensis (Coleoptera, Dytiscidae)
}

Ph. Richoux ${ }^{1}$

J. Dalmon ${ }^{2}$

J.L. Reygrobellet ${ }^{1}$

Mots clés : Coleoptera, Dytiscidae, phréatobie, Siettitia, France.

La découverte d'une nouvelle station de Sietritia avenionensis dans la région d'Avignon a pernis de préciser le statut taxonomique de cette espèce phréatobie et de confïrmer sa présence dans toute la vallée du Rhône. Le suivi de la population de cette station durant 9 mois confirme les hypothèses émises précédemment, d'après l'étude d'une population lyonnaise, concernant son cycle biologique (espèce monovoltine à développement larvaire estival et automnal) et les effets des mouvements de nappe sur ses déplacements dans l'aquifère.

Additional information on the systematics and ecology of Siettitia avenionensis (Coleoptera, Dytiscidae).

Keywords : Coleoptera, Dytiscidae, Stygobiont, Siettitia, France.

The discovery of a new locality for Siettitia avenionensis in the Avignon region has facilitated a more precise evaluation of the taxonomic status of this stygobiont species and has confirmed its presence throughout the Rhône valley. A study of the population at this locality over 9 months confirmed a previous hypothesis from a study of a Lyon population with respect to the life cycle (monovoltine species with larval development in the summer and autumn) and the effects of fluctuations in water level on their movements within the aquifer.

\section{Introduction}

Siettitia avenionensis est une espèce phréatobie décrite par Guignot en 1925 sur un scul exemplaire trouvé dans un puits en Avignon. En 1976, des individus furent capturés dans la plaine alluviale du Rhône en amont de Lyon à Balan (Gibert et al. 1977) ; ils furent considérés comme appartenant à l'espèce $S$. avenionensis et le mâle fut décrit (Richoux 1978). L'importance de cette population lyonnaise, étudièe durant plusieurs années par pompage dans la nappe phréatique, avait permis de

1. Laboratoire de Biologie animale et Ecologie. URA CNRS 367. Université Claude Bernard, Lyon 1. F,69622 Villeurbanne cédex.

2. 21, rue du Doyen Gosse. F. 38700 La Tronche. définir le cycle de développement avec trois stades larvaires anophtaimes (Richoux 1980) et de donner quelques premières informations écologiques (Richoux \& Reygrobellet 1986). Malheureusement cette station est détruite. La capture d'un exemplaire dans les gorges de l'Ardèche par Vervier (1988) nous permettait d'espèrer retrouver cette espèce dans d'autres stations du bassin du Rhône.

En août 1989, l'un d'entre nous (J.D.) a découvert des adultes de Siettitia dans une nouvelle station très proche de la station du type. Nous avons alors pensé que l'étude des exemplaires capturés nous permettrait de vérifier le biẹn-fondé de la détermination des exemplaires de la région lyonnaise.

Une campagne de prélèvements de faune, en particulier des différents stades de Siettitia, a été menée à partir d'octobre 1989, afin de poursuivre les 
études écologiques entamées sur la population lyonnaise et de comparer les résultats. Simultanément des investigations sur l'hydrologie de la station ont débuté.

\section{Situation et description de la station}

Cette nouvelle station correspond à un puits situé dans la commune de Barbentane (Bouches-duRhône). Elle se situe, à moins de $10 \mathrm{~km}$ au sud de la localité d'Avignon (Vaucluse), sur la rive gauche du Rhône en aval de sa confluence avec la Durance. Ce puits se trouve au pied de l'affleurement rocheux du Massif de la Montagnette (calcaire Miocène) en bordure de la plaine alluviale au lieu-dit « Le Trouillet $"$. Le niveau d'eau de ce puits (environ 3,5 m de profondeur) est pratiquement constant malgré les fortes sécheresses de 1989 et 1990 et les fluctuations printanières de la nappe alluviale, ce qui suggère une arrivée d'eau souterraine en provenance de la Montagnette.

Afin de vérifier cette hypothèse une étude physicochimique préliminaire a été menée le 13 août 1990. Pour cela nous avons prélevé et analysé les eaux de cette station, d'un autre puits situé à Barbentane dans la Montagnette au lieu-dit "Les Carrières". de la Durance à Rognonas (Bouches-du-Rhône) e: du Rhône en amont de la confluençe en rive droit aux Angles (Gard) (fig. 1).

Les résultats (Tableau I) montrent sans ambigüité que les eaux des deux puits n'ont aucun rapport avec les nappes alluviales à cette époque de l'année, que ce soit celle de la Durance ou celle du Rhône. Les eaux de ces puits, caractéristiques d'écoulements karstiques, sont relativement similaires $(\mathrm{pH}, \mathrm{SiO} 2$, TAC) ; cependant, quelques différences existent

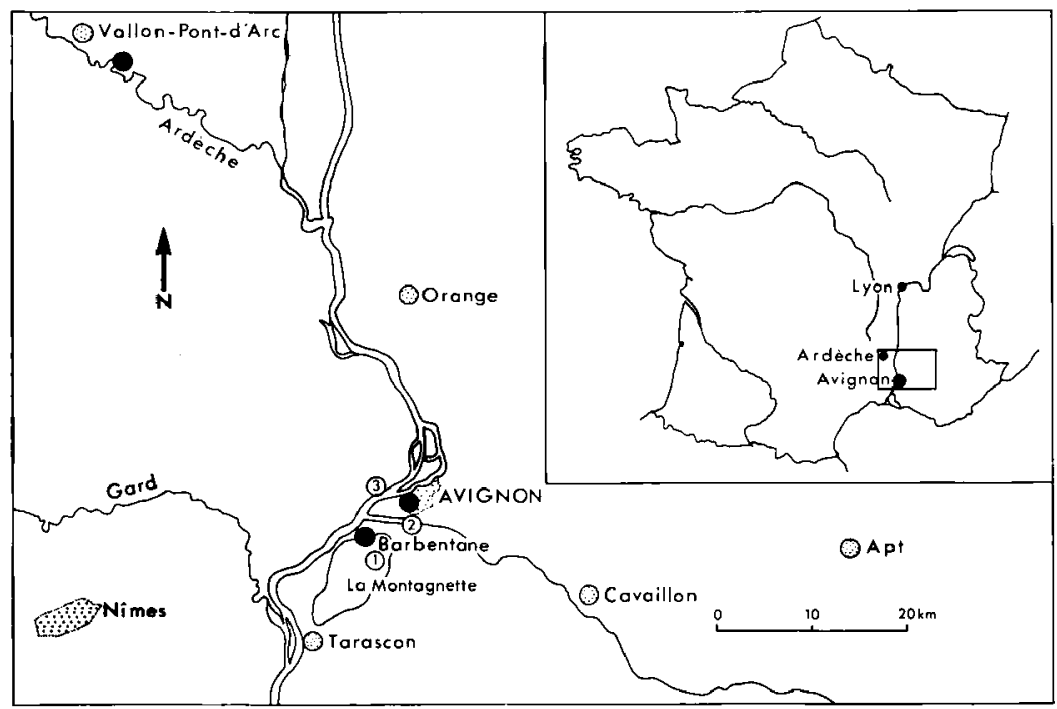

Fig. 1. Cartes des stations de Siettitia avenionensis ( $O$ ) et des stations de mesures physico-chimiques : $1:$ Barbentane (puits des « Carrières " ; 2 : Rognonas (La Durance) ; 3 : Les Angles (Le Rhône). 


\begin{tabular}{|c|c|c|c|c|c|c|c|c|c|c|}
\hline Stations & pH & $\begin{array}{l}\mathrm{SiO} 2 \\
\mathrm{mg} / \mathrm{I}\end{array}$ & $\begin{array}{l}\text { TAC } \\
\mathrm{mg} / 1\end{array}$ & $\begin{array}{c}\mathrm{Ca} \\
\mathrm{mg} / \mathrm{I}\end{array}$ & $\begin{array}{l}\mathbf{M g} \\
\mathbf{m g} / \mathrm{I}\end{array}$ & $\begin{array}{c}\mathrm{Cl} \\
\mathrm{mgl}\end{array}$ & $\begin{array}{l}\mathrm{SO} 4 \\
\mathrm{mg} / \mathrm{I}\end{array}$ & $\begin{array}{l}\mathrm{NO} 3 \\
\mathrm{mg} / 1\end{array}$ & $\begin{array}{c}\text { conductlvite } \\
\mu \mathrm{S}\end{array}$ & $\begin{array}{c}\text { tomperalure } \\
\text { c }\end{array}$ \\
\hline $\begin{array}{c}\text { Barbentane } \\
\text { (pults) } \\
\text { Le Trouillet }\end{array}$ & 6,99 & 17,5 & 307,5 & 134,7 & 9,2 & 22,5 & 50 & 6,4 & 696 & 15,1 \\
\hline $\begin{array}{c}\text { Barbentane } \\
\text { (puits) } \\
\text { Les Carrieres }\end{array}$ & 7,05 & 22,5 & 306,3 & 173,2 & 18,9 & 24,5 & 190 & 4,1 & 1042 & 15,8 \\
\hline $\begin{array}{l}\text { Rognonas } \\
\text { (Durance) } \\
\text { pont N570 }\end{array}$ & 7,45 & 3,8 & 132,5 & 78,2 & 9,7 & 27,5 & 80 & 0,9 & 485 & 23,4 \\
\hline $\begin{array}{c}\text { Les Angles } \\
\text { (Rhone) } \\
\text { Grange-Neuve }\end{array}$ & 7,83 & 1,75 & 96,2 & 57,3 & 1,5 & 20,5 & 42 & 1,6 & 335 & 24,8 \\
\hline
\end{tabular}

Tableau I. Mesures physico-chimiques du 13 aoút 1990 de l'eau des 4 stations définies par la commune, le type d'eau prélevée, le lieu-dit.

entre elles pour les teneurs en $\mathrm{Ca}$ et $\mathrm{Mg}$ et donc pour la Conductivité. Ces différences seraient dues au fait que le puits des "Carrières " n'est plus en usage actuellement à l'inverse de celui du "Trouillet". Des recherches complémentaires sont prévues afin de mieux établir, à différentes périodes du cycle hydrologique, l'origine des eaux de ces deux puits ainsi que des puits voisins cernant le massif de la Montagnette.

Cette station semble donc, comme celle de Balan (Richoux \& Reygrobellet 1986), correspondre à un aquifère latéral confluant avec une nappe alluviale.

\section{Statut de l'espèce Siettitia avenionensis Guignot, 1925}

Le genre Sietritia Abeille de Perrin comprend deux especes, toutes deux françaises: $S$. balsetensis Abeille de Perrin, 1904, récoltée en deux stations du département du Var actuellement détruites et $S$. avenionensis décrite sur l'unique exemplaire femelle trouvé par Guignot (1925) en Avignon.

En 1976, des individus de Siettitia furent capturés dans la plaine alluviale du Rhône sur la commune de Balan (Ain). Après comparaison avec les exemplaires de Siettitia de la collection Guignot au Muséum national d'Histoire naturelle de Paris, y compris le type de $S$. avenionensis, il a été admis que la population lyonnaise devait être rattachée à l'espèce $S$. avenionensis et en conséquence le mâle fut décrit (Richoux 1978). Cependant un doute subsistait : les individus lyonnais n'appartiendraient-ils pas à une troisième espece ?

Vu la proximité de la localité-type, il est logique d'admettre l'appartenance des individus de la station de Barbentane à l'espèce $S$. avenionensis d'autant plus que les femelles ne présentent aucune différence avec le type.

L'observation des mâles nous permet done de compléter la description de l'espèce et de les comparer avec les individus de la région lyonnaise.

D'un point de vue morphologique, les individus sont totalement conformes aux descriptions de Guignot (1925, 1931-33) et de Richoux (1978). L'édéage (fig. 2) correspond en tous points à celui des individus lyonnais (Richoux 1978) ; seul l'apex des paramères semble un peu moins proéminent, la courbure vers l'intérieur de cet apex variant en fonction du degré de déshydratation de ces pièces génitales. Ces paramères restent cependant très différents de ceux de $S$. balsetensis (Guignot 1931-33) avec leur apex émoussé, un bord dorsal régulièrement courbe et une apophyse basale plus longue.

En conséquence on peut maintenant admettre sans équivoque que $S$. avenionensis est une bonne espèce, différente de $S$. balsetensis et que son aire de répartition s'étend, dans l'état actuel de nos connaissances, le long de la vallée du Rhône (fig, 1). 

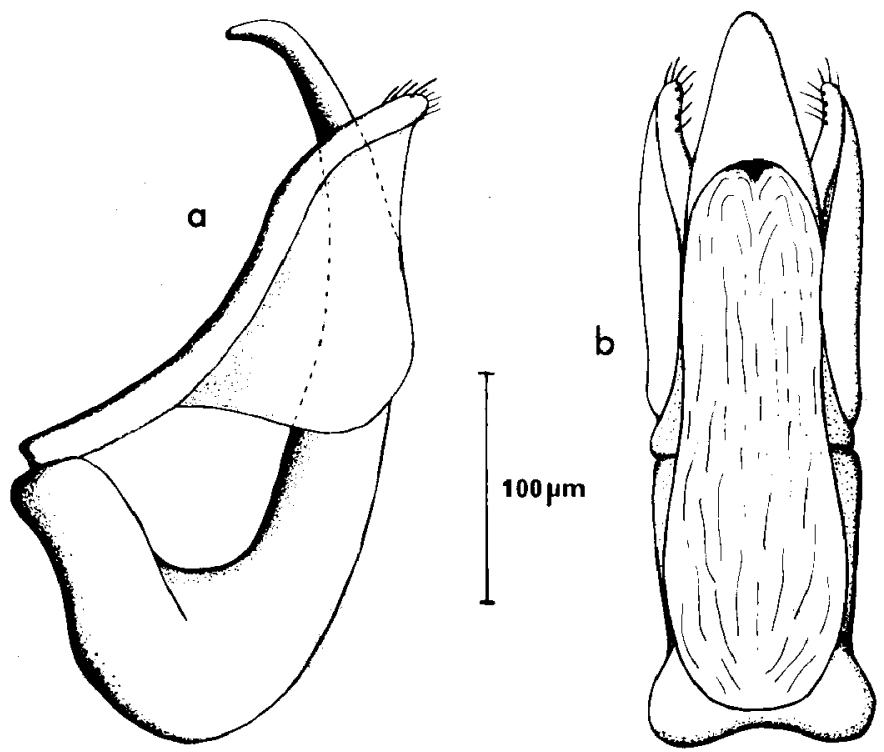

Fig. 2. Edéage du mâle de Siethitia avenionensis de Barbentane. a) vue de profil : pénis et paramère droit. b) vue de dessus : pénis et paramères.

\section{Dynamique de population de Siettitia avenionensis}

Après la découverte d'adultes de Siettitia en août 1989 , des prélèvements de faune ont été effectués à l'aide d'une pompe manuelle à clapet en place dans le puits du «Trouillet ". Ce puits profond de $5 \mathrm{~m}$ a une épaisseur d'eau d'environ 1,5 m. L'une des parois correspond à la roche mère, les autres étant construites. La crépine de la pompe est située contre la paroi rocheuse tout près du fond, les prélèvements ne récupérant piatiquement pas de sédiment. La faune est récoltée sur un tamis de $160 \mu \mathrm{m}$ ce qui permet de capturer les jeunes larves de $S$. avenionensis. Les prélèvements se sont échelonnés, à partir d'octobre 1989, avec une périodicité de l'ordre de 3 à 4 semaines par séries de 2 à 10 pompages de 50 à 150 litres sur 1 à 8 jours. A partir du mois de mars, les séries ne se composent que de pompages quotidiens de $100 \mathrm{l}$. Afin d'homogénéiser les résultats, nous ne tiendrons compte que des densités d'individus par série ramenées à un volume de $100 \mathrm{l}$.

La figure 3 montre les histogrammes de fréquence des différents stades de $S$. avenionensis : adulte, stades larvaires I et III (les stades II n'ont pas été rencontrés) durant la période de récolte du 7 octobre 1989 au 25 juillet 1990. Les adultes sont en densité beaucoup plus importante que les larves ( 1 à 7 adultes contre seulement 0 à 1,7 larves pour $100 \mathrm{l}$ d'eau pompée). Pour les adultes, on remarque une relative stabilité de la population tout au cours du cycle de pompage (la densité variant globalement entre 2 et 4) avec une augmentation nette en janvier ( $d=$ 7). Les larves de stade III sont présentes, en faible 

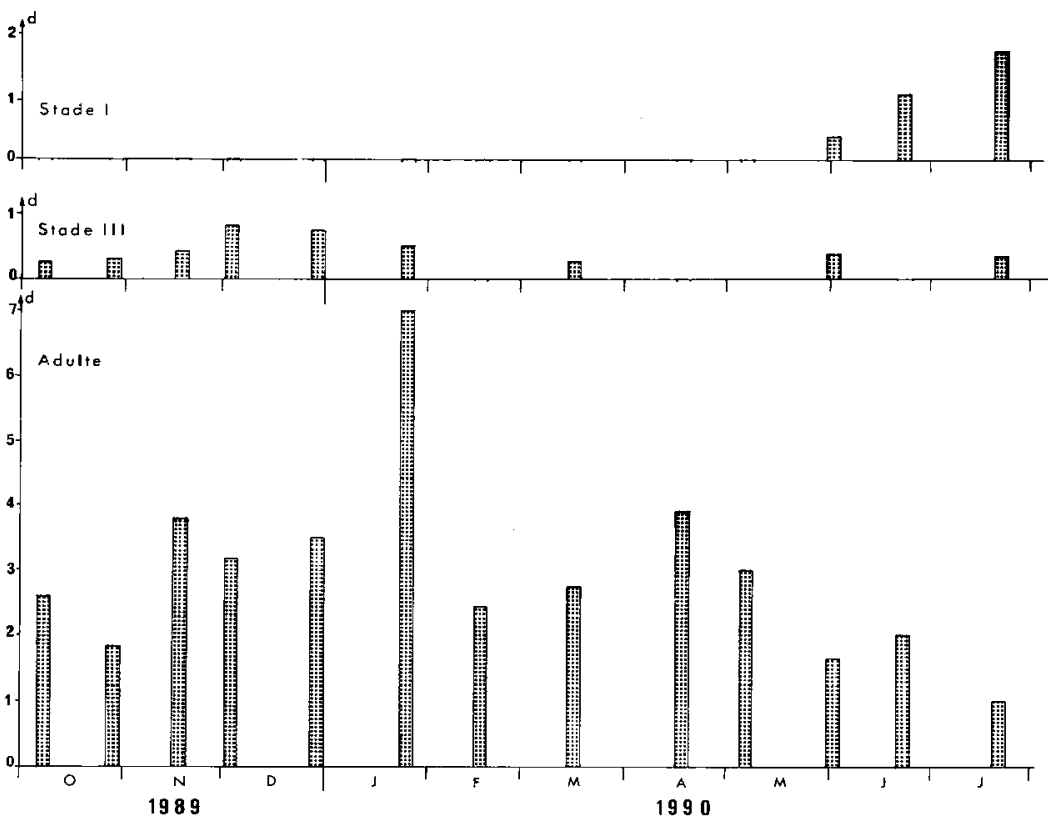

Fig. 3. Histogrammes de densité (d) des adultes et des stades larvaires par $100 \mathrm{l}$ d'eau durant la période d'octobre 1989 à juillet 1990.

densité $(0$ à 0,8$)$, tout au long de nos premières investigations. Enfin il est intéressant de remarquer l'apparition des stades I au début de l'été.

La comparaison de ces résultats avec ceux de la région lyonnaise montre que, dans cette station à très faible battement de nappe, le cycle de développement correspond avec le modèle hypothétique proposé par Richoux \& Reygrobellet en 1986 ; développement larvaire en été et automne avec, toute l'année, présence de quelques stades III ; nymphose et mue imaginale en hiver. D'autre part, cette étude nous conforte dans l'idée que les variations de nappe ont un rôle important dans les déplacements des populations à l'intérieur de l'aquifère, comme cela était le cas pour la population de la zone de Balan où de brusques fluctuations de niveau entraînaient de fortes variations positives ou négatives de l'abondance de $S$. avenionensis en un lieu donné.

\section{Conclusions}

La découverte de cette nouvelle station de $S$. avenionensis nous a d'abord permis de confirmer le statut de cette espèce et sa répartition géographique.

L'étude préliminaire de cette population avignonnaise nous indique que les hypothèses formulées à partir des données obtenues sur la population lyonnaise s'avèrent correctes : modèle de cycle biologique et effet des variations de nappe.

Profitant des possibilités d'accès à un aquifère à faibles variations de niveau, où les risques de destruction semblent actuellement très limités, nous pensons pouvoir suivre régulièrement cette population 
de $S$. avenionensis durant plus d'une année afin d'approfondir l'étude de son cycle biologique. Simultanément, l'évolution des peuplements des autres macroinvertébrés (essentiellement des Annélides et des Crustacés) sera suivie en fonction des variations limitées de l'aquifère local et des fluctuations plus importantes de la nappe alluviale toute proche. Ces recherches devraient nous permettre, grâce à la comparaison des peuplements lyonnais et avignonnais, de mieux cerner les problèmes écologiques liés au contact d'une nappe alluviale avec un aquifère latéral.

\section{Travaux cités}

Gibert (J.), Ginę (R.,) Mathieu (J.), Reygrobellet (J.L.) \& SeyedReihani (A.). 1977. - Structure et fonctionnement des écosystèmes du Haut-Rhône français. IV. Le peuplement des eaux phréatiques, premiers résultats. Annis Limnol. 13 (1) : 83-97.
Guignot (F.). 1925. - Description d'un Siettitia nouveau du Midi de la France (Col. Dytiscidae). Bull. Soc. ent. Fr. 2 : 23-24.

Guignot (F.). 1931-33. - Les Hydrocanthares de France. Ed. Miscel, entom. Toulouse : 1057 p.

Richoux (Ph.). 1978. - Description du mâle de Sietitia avenionensis : Coleoptère Dytiscidae phréatobie trouvé dans la région lyonnaise. Bull. mens. Soc. linn. Lyon 47 (7) : 389-392.

Richoux (P.). 1980. - Les larves du Coléoptère Dytiscide phréatobie: Siertitia avenionensis Guignot. Mém. Biospéol. 7 : 195-200.

Richoux (Ph.) \& Reygrobellet (J.L.). 1986. - First report on the Ecology of the Phreatic Water Beetle Siettitia avenionensis Guignot (Coleoptera, Dytiscidae). Entomologica Basiliensia $11: 371-384$.

Vervier (P.). 1988. - Hydrologie et dynamique des peuplements aquatiques souterrains : Comparaison de deux systèmes karstiques des Gorges de l'Ardèche. Thèse Université ClaudeBernard, Lyon I : 219 p. 